\title{
Hard and soft osteo-arthritis
}

\author{
C. P. BRoAD \\ M. B., F.R.C.S. \\ The Rowley Bristow Orthopaedic Hospital, Pyrford, Surrey
}

\section{Summary}

This short paper attempts to show how the $X$-ray appearance influences the choice and success of operative procedures performed on the hip and knee joints for osteo-arthritis. If the bones look 'soft' the only methods likely to succeed are those in which total replacement coupled with the use of cement ensure firm anchorage and wide distribution of stress. When bones look 'hard' simpler methods are usually satisfactory.

IN THE treatment of osteo-arthritis it is usually easy to decide whether or not operative treatment is indicated: but frequently it is difficult to choose which operation is best suited to the individual patient. The three basic procedures are arthrodesis, osteotomy and arthroplasty. Arthrodesis is usually reserved for those patients in whom one joint only is involved, the "compensating' joints are reasonably supple and the patient fairly young. Osteotomy is most likely to succeed where the joint has a reasonable range of movement and the disease is not so advanced that bone collapse precludes the probability of joint regeneration. Arthroplasty is the only feasible procedure for all other patients and nowadays a variety of techniques is available.

The present hypothesis is not concerned with those cases selected for arthrodesis. It attempts to rationalize the choice between osteotomy and arthroplasty as well as indicating which technique of arthroplasty should be used.

The hip and knee joints were selected for study, these being the joints at which the problem was most often encountered. When patients were examined at least 2 years after operation it was relatively easy to decide which were successful or which were failures. Next the $X$-ray films were studied and again two clear-cut groups emerged; those in whom the bones looked 'hard' (Fig. 1a) and those in whom they looked 'soft' (Fig. 1b). The third stage of the investigation was to try and correlate success or failure with hardness and softness of bone. The thesis of this article is that such a correlation not only exists but is remarkably constant and has a rational basis in pathology.

Secondary osteo-arthritis may follow mechan- $\vec{\circ}$ ical anomalies (e.g. fractures into the joint, devel- $\vec{\omega}$ opmental disorders, mal-alignment, etc.) in which case the texture of the bone is relatively normalo and it looks 'hard' on X-ray. But when the degen- 3 erative changes are secondary to rheumatoid $\vec{f}$ arthritis the bone looks 'soft', as it also does in $\sigma$ osteoporotic disorders.

When 'soft' bone osteoarthritis of the hip was treated by prosthetic replacement of the femoral head the result was often unsatisfactory. Pain wais not relieved or it subsequently recurred ; and late $\vec{Z}$ $\mathrm{X}$-ray films showed the prosthesis migrating dow the femoral shaft, or burrowing into the pelvis, of both (Fig. 2). Even osteotomy rarely succeeded presumably because any biological regeneration was more than offset by the continuing collapse $\vec{\varphi}$ of the soft bone. But even with 'soft' bone total hip-replacement using McKee's techniqu世 was nearly always successful. The essential feature is probably the wide distribution of stress afforded by the use of the cement which anchors the metal components.

With 'hard' bone osteo-arthritis at the hip, $\stackrel{\mathbb{Q}}{\square}$ procedures less elaborate than total replacement $\overline{\vec{\sigma}}$ were usually adequate. Osteotomy was successful (providing the joint preoperatively had at least $70^{\circ}$ of flexion and little bone collapse had occurred), and cup arthroplasty or simple replacement of the femoral head with a Moore's or Thomson's prosthesis were also satisfactory. Similarly Ring's total hip replacement although not employing cement was successful, presumably because the long screw carrying the acetabular component obtained an excellent grip on the 'hard' bone of the ilium.

At the knee the findings were similar. With 'soft' bone osteo-arthritis neither osteotomy nor $N$ simple tibial plateau replacement proved reliable; $N$ continuing collapse caused failure and success could be obtained only by using total knee replacement in which each component was anchored with cement distributing the load widely. When the bone was 'hard' osteotomy or 


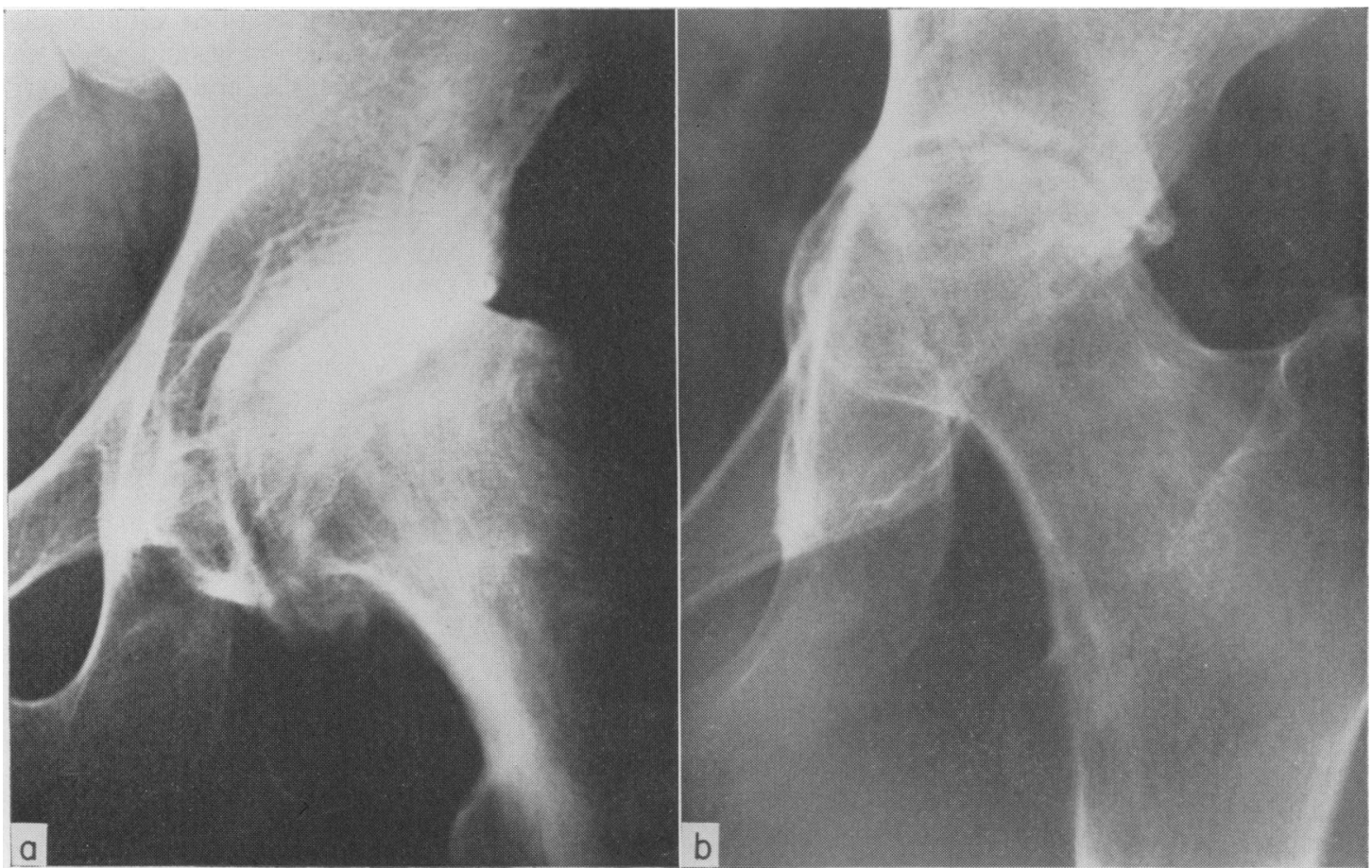

FIG. 1. 'Hard' (a) and 'soft' (b) osteo-arthritis.

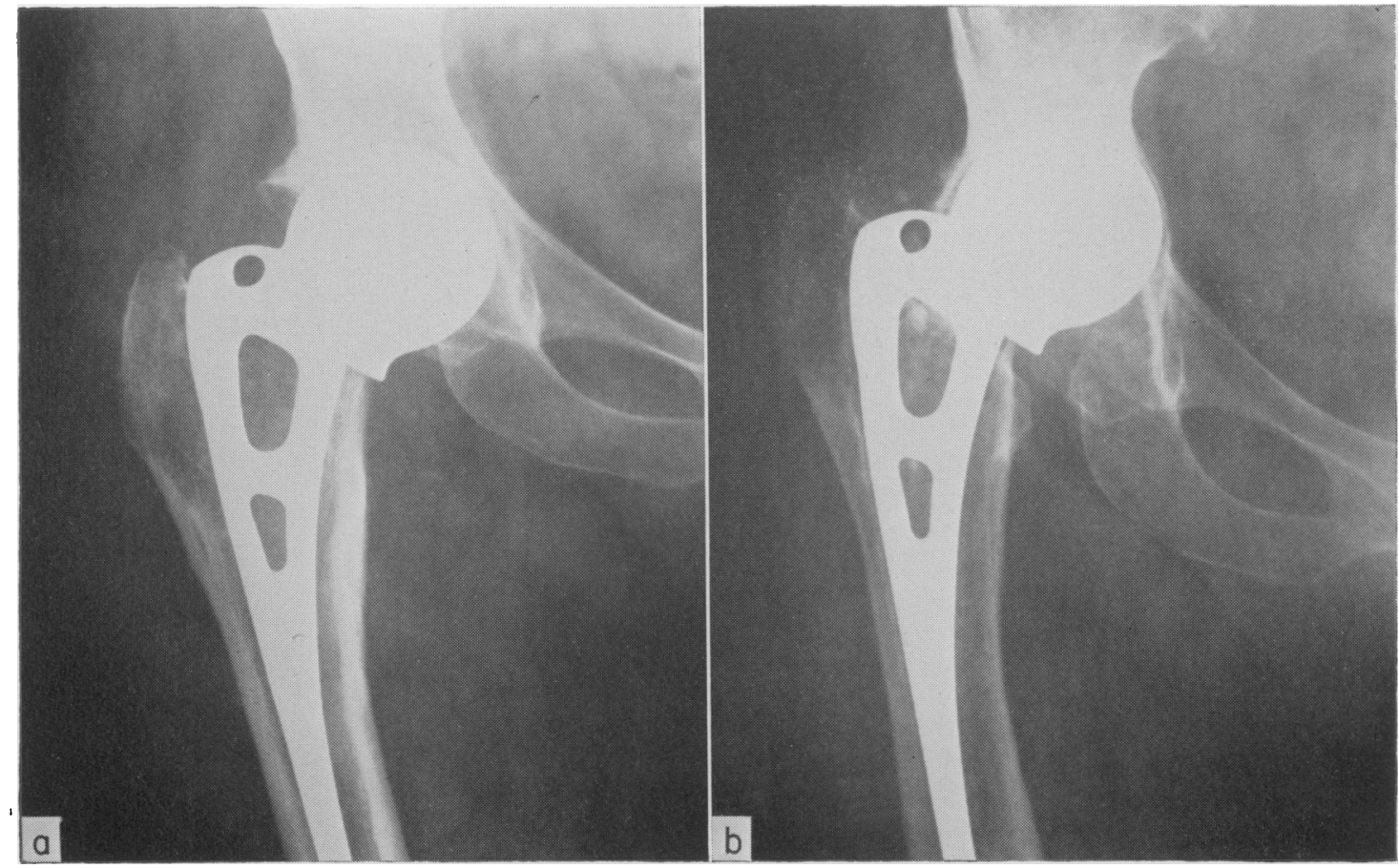

FIG. 2. (a) A Moore's prosthesis which has been inserted in the presence of 'soft' bone. (b) The same hip as illustrated in (a) after an interval of 3 years. The prosthesis has sunk down the shaft of the femur and into the acetabulum. 
tibial plateau prosthetic replacement were usually satisfactory.

\section{Conclusions}

In choosing between the various procedures available in the surgical treatment of osteo-arthritis the radiographic appearance of the bones is of considerable importance. If the bones look 'soft' the only methods likely to succeed are those in which total replacement coupled with the use of cement ensure firm anchorage and wide distribution of stress. When the bones look 'hard' simpler methods are usually satisfactory.

\section{Acknowledgment}

I wish to thank Mr A. Graham Apley who encouraged me to develop this thesis. 